\title{
Flying Ad hoc Networks (FANET): Performance Evaluation of Topology Based Routing Protocols
}

\author{
https://doi.org/10.3991/ijim.v16i04.28235 \\ Ali H. Wheeb ${ }^{(凶)}$ \\ Faculty Member - Lecturer, University of Baghdad, Baghdad, Iraq \\ a. wheeb@coeng. uobaghdadedu.iq
}

\begin{abstract}
Flying Ad hoc Networks (FANETs) has developed as an innovative technology for accessing places without permanent infrastructure. This emerging form of networking is construct of flying nodes known as unmanned aerial vehicles (UAVs) that fly at a fast rate of speed, causing frequent changes in the network topology and connection failures. As a result, there is no dedicated FANET routing protocol that enables effective communication between these devices. The purpose of this paper is to evaluate the performance of the category of topology-based routing protocols in the FANET. In a surveillance system involving video traffic, four routing protocols with varying routing mechanisms were examined. Additionally, simulation experiments were conducted to determine the influence of flying altitude. The results indicate that hybrid routing protocols outperform other types of protocols in terms of average throughput. Proactive protocols, on the other hand, have the least jitter.
\end{abstract}

Keywords - multi-UAV, flying ad hoc networks, topology-based routing protocol, Gauss Markov, flying altitude

\section{Introduction}

Unmanned aerial vehicles (UAVs) have made significant advancements and are now extensively utilize, whereas wireless data transfer technologies have indeed made significant advancements. All of this contributes to the emergency communications system types. The Flying Ad-Hoc Network is one of these communication networks (FANET) [1]. FANET is an autonomous self-organizing network. Its nodes not only link to their neighbors, but they also relay traffic via them [2]. Only a subgroup of UAVs may communicate with a satellite or ground station and all flying UAVs form an ad-hoc network. Thus, in addition to the base station, the UAVs may communicate with one another. FANET has several advantages, including adaptability, increased accuracy, economy, continuity, Flexibility, and speed [3] [4].

FANETs are becoming more and more popular with each passing day. Previously, FANETs are basic remotely controlled airplanes that have been primarily employed in military operations [5]. Nevertheless, in recent years, FANETs have been deployed in a growing variety of civil and commercial activities, including search and rescue [6], package delivery [7], traffic monitoring in smart cities [8], agriculture [9], engineering 
education [10], mobile drone in indoor localization [11] and disaster monitoring [12]. The application of FANET is depict in Figure 1.

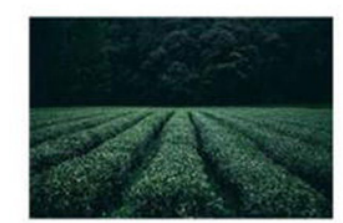

Monitoring of agricultural areas

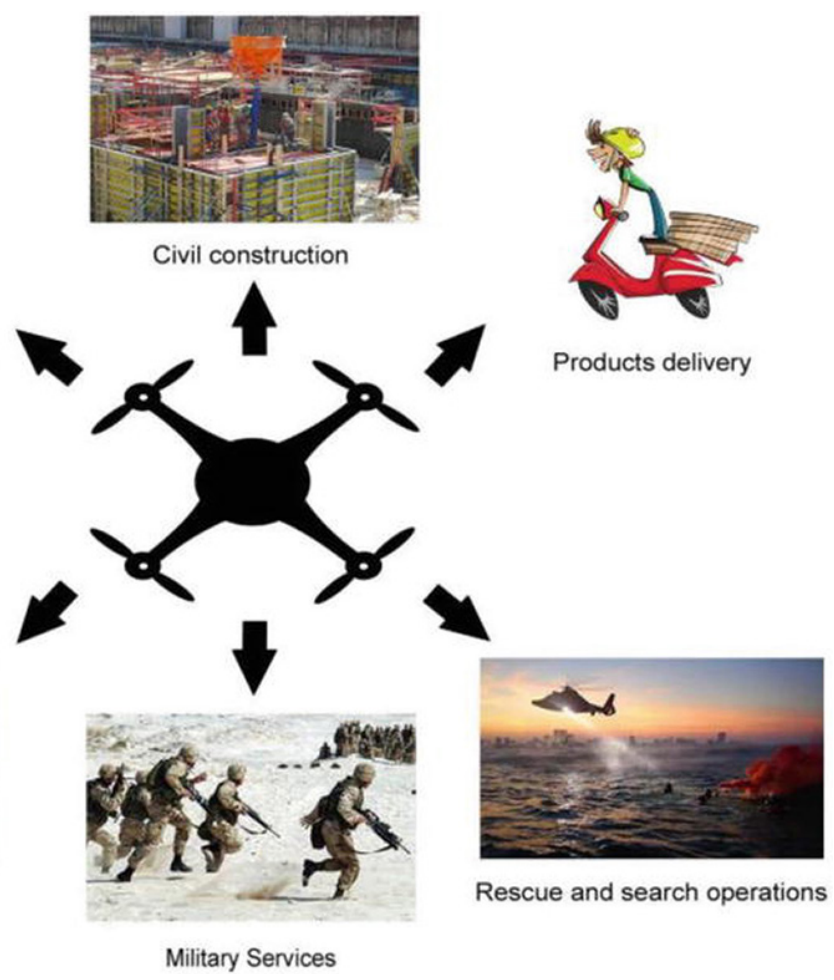

Fig. 1. Applications of FANET

The degree of mobility of FANET nodes is significantly higher than that of VANET or MANET nodes. Because of the high mobility of FANET nodes, the topology of the network changes on a more regular basis. FANET has many unique problems, with the routing process being one of the most significant design considerations. To function properly in FANET, the routing protocols used must be capable of performing an automated search for the optimum route to offer one or more subjective parameters for the operation of data transmission and receiving [13].

The primary goal of this paper is to define FANET as a unique ad hoc network family and to assess the effectiveness of topological routing protocols in FANET. This article makes three contributions: (i) presenting various routing challenges; (ii) categorizing current topology-based routing protocols in FANET; and (iii) comparing and analyzing them using various performance measures. Our comparison study will aid network engineers in selecting optimal routing protocols for the FANET deployment scenario. 
The remainder of the paper is laid out as follows: Section 2 discusses FANET routing problems as well as the taxonomy of topology-based routing systems. Describe the simulation setup and the mobility model in Section 3. The results of the analysis will be provided in section 4 , followed by a discussion. The conclusions are in Section 5.

\section{Routing in FANET}

\subsection{Challenge of routing protocols in FANET}

Routing protocols are in charge of discovering, creating, and maintaining communication routes between two nodes. The overhead and bandwidth usage of these protocols should be kept to a bare minimum. Due to the high mobility of UAVs, network topology might vary over time, making route finding and route maintenance one of the most important challenges to solve [14]. To enhance routing performance, including better QoS and high route setup success ratio, as well as lower energy consumption. There are three major issues to overcome [15]:

High network dynamicity. Due to the obvious high mobility of FANETs, the extremely dynamic network topology results in low connections between nodes. as a result, and network partitions and link disconnections are common, increasing route discovery and maintenance and lowering routing performance [15]. To investigate the routing process in FANET, many UAV mobility models have been developed [16].

Residual energy. According to the comparably large distance between UAVs, UAVs powered by batteries have limited energy (a) to conduct routing processes such as route discovery, updates, and maintenance; (b) to provide extended transmission range; (c) retransmit various packets when a link failure occurs. UAVs carrying heavier payloads, on the other hand, consume more energy [17].

High resource costs. Frequent route discovery, updates, reestablish routes, and different packet retransmissions in FANETs can result in three categories of resource costs: (a) high routing overhead or inefficient bandwidth usage); (b) computational cost owing to route processing time; (c) excessive energy consumption. To improve UAV connectivity, multi-UAVs can be deployed in many scenarios [18].

\subsection{Topology based routing protocols in FANETs}

FANET routing protocols, as shown in Figure 2, can be classified into four groups based on the approach used and the challenges that must be overcome. Topology-based routing protocols, which are based on link information, use IP addresses to exchange packets between interacting nodes. This category includes four types: static, proactive, reactive, and hybrid [19]. The following section delves into the topology-based routing protocols category and its most important routing protocols. 


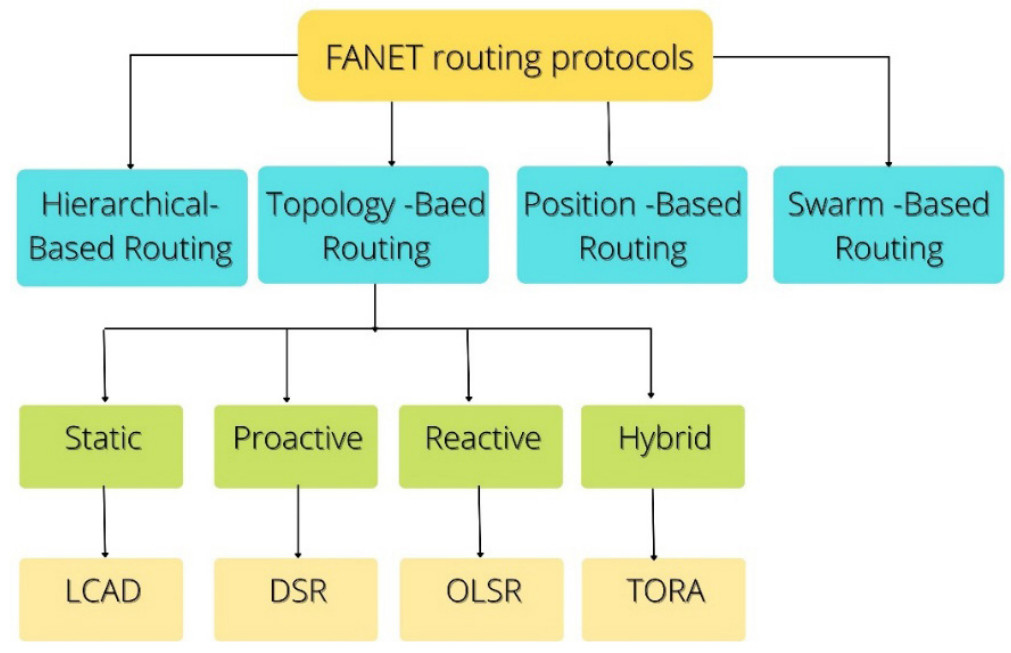

Fig. 2. Taxonomy of topology based routing protocols in FANET

In static protocols, the information for UAVs is calculated and loaded to each UAV first in this type of routing protocol. It is not possible to modify it during the process, and the network topology should always be fixed. As a result, the number of communication lines between UAVs or between the UAV and the ground station is reduce. This routing technique does not offer fault tolerance in a dynamic environment if certain UAVs fail since they must wait until the mission is over to rectify the issue.

Load Carry and Deliver (LCAD) [20] is a FANET-specific static routing protocol. Before UAVs take off, LCAD configures the navigation path on the ground. UAVs are thought of as connections between a source and a destination ground control station, collecting packets of data, transporting them, and transmitting them to the destination. If the UAVs carrying the packets of data are not heading in the proper direction, other UAVs might take over and deliver the data packets. Figure 3 illustrates the LCAD routing technique in a FANET. It must be noted that no routing table method is used in this technique. 


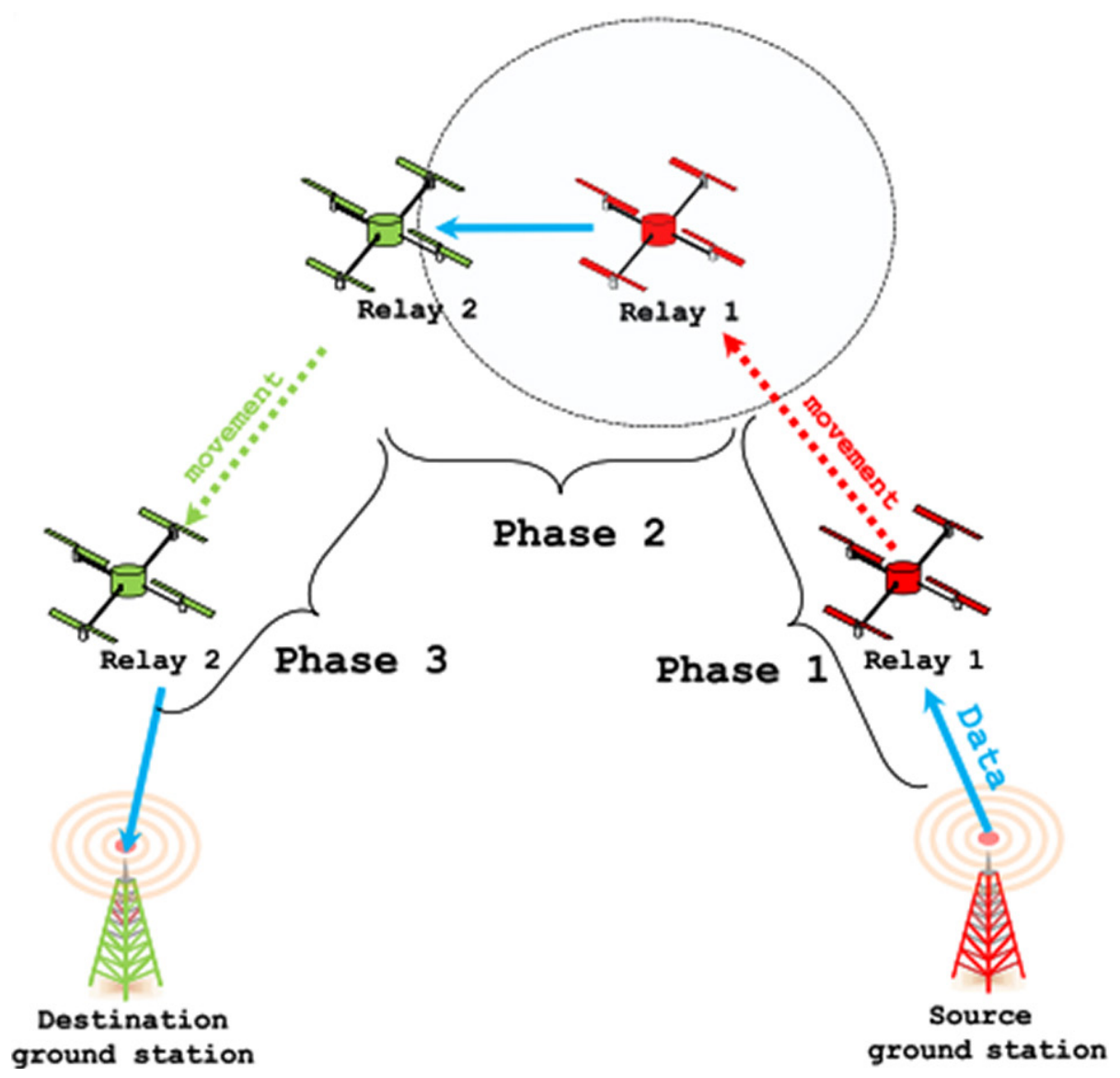

Fig. 3. LCAD mechanism in FANET

In reactive protocols, the path between nodes is establish when there is a request to transmit packets. When a request to transmit packets is receive, a path between nodes is construct in reactive protocols. As a result, there is no need to estimate the routes for each node regularly. It appears to resolve the overhead issue. There are two different kinds of messages in this mechanism: route request and route reply. The key benefit of this approach is its bandwidth efficiency. However, it will be delayed due to the time required to identify the path [21].

Dynamic Source Routing (DSR) [22] is a reactive routing technology that enables a network to self-organize and customize themselves without the use of infrastructure. Since DSR is reactive, a discovery procedure is only used when information is transmitted. A route maintenance system is also used to keep track of any path failures. DSR's flexibility of loop characteristics allows users to choose from numerous routes to any target node. Because each transmitted packet must include all of the transited nodes' addresses, it is insufficient for large-scale networks and networks. Figure 4 depicts the DSR mechanism for FANET. 


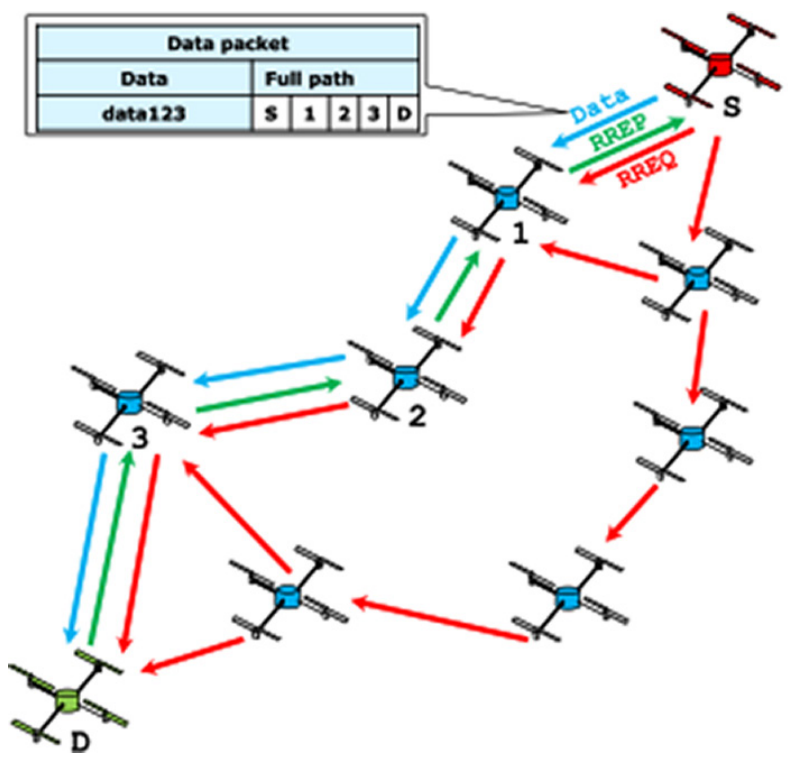

Fig. 4. DSR mechanism in FANET

In proactive protocols, the table-based routing contains all of the information about all nodes within the network, allowing each node to know everything there is to know about the others in the network. This approach has one major benefit: each node's table continuously contains the most up-to-date information about another node. However, we must keep in mind that this technique requires bandwidth due to the cost of the updated messages for the tables. Due to the limited bandwidth available in the FANETs network, customized routing protocols can be utilize to modify the topology of the nodes.

Optimized Link State Routing Protocol (OLSR) [23] [24] is a link-state routing protocol that establishes a global knowledge of all current UAV-to-UAV connections. This is accomplish by periodically exchanging Hello and Topology Control (TC) packets between the UAVs to update the network's topology information. OLSR chooses MultiPoint Relay (MPR) UAVs to cover two-hop neighbors, produce link-state information, and relay data packets to other MPRs, lowering overhead. The OLSR mechanism in FANET displays in Figure 5.

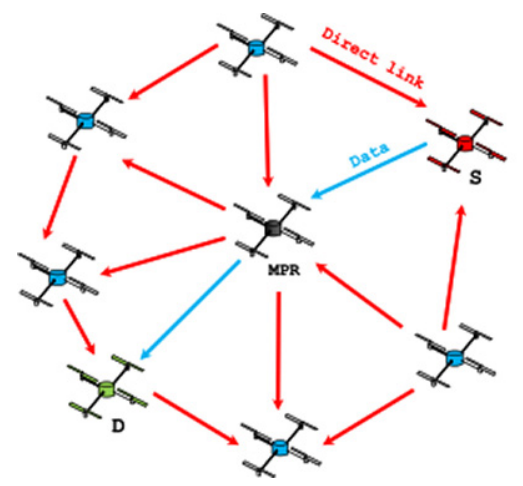

Fig. 5. OLSR mechanism in FANET 
Hybrid protocols integrate the reactive and proactive aspects of protocols. It was created with the goal of reducing overhead and maximizing bandwidth use. As a result, hybrid protocols are appropriate for large-scale networks with many sub-network regions, where intra-zone routing employs proactive mechanisms and inter-zone routing employs reactive mechanisms. Hybrid protocol adopted in many FANET Application [25].

Temporarily Ordered Routing Algorithm (TORA) [26] is a hybrid distributed routing technique that performs well in highly dynamic networks such as FANETs. TORA is exclusively responsible for updating and maintaining the communication links between nearby UAVs. TORA's major goal is to minimize the number of control packets sent during topology changes. TORA constructs and maintains a Directed Acyclic Graph (DAG) between communicating UAVs that have several paths. Furthermore, TORA frequently chooses longer routes in order to save overhead. To summarize, TORA employs both reactive and proactive techniques depending on the network's state, and it discovers alternate routes in the event of connection failures. Figure 6 depicts the TORA mechanism in FANET.

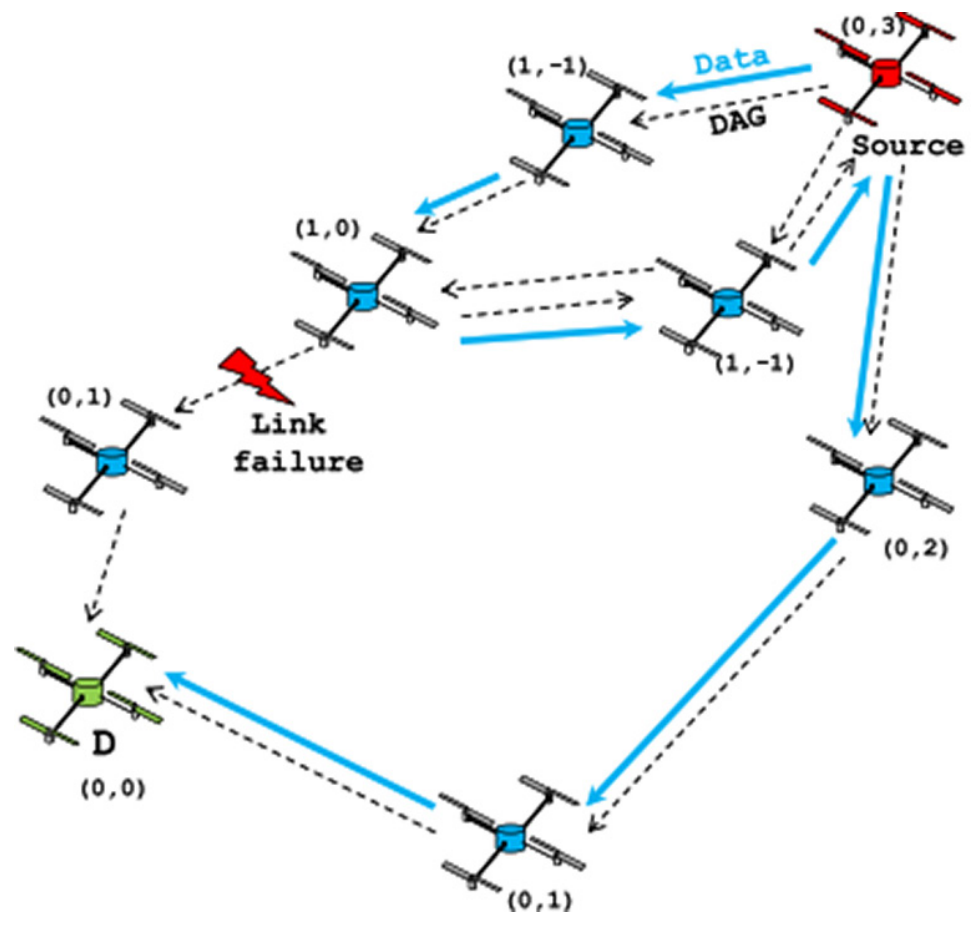

Fig. 6. TORA mechanism in FANET

\section{Simulation set up}

Due to the high cost of real UAVs and the time and resources required to create a realistic FANET environment, the network simulator was used to deploy the UAVs and facilitate communication among them. In the simulation challenge, a network 
environment that is as near to the real world as possible must be suggested [27]. In light of this, researchers should attempt to build realistic FANETs application situations.

\subsection{Simulation of FANET}

A single source UAV node 1 , a single base station node 20 , and 18 relaying UAV nodes make up the FANETs simulation scenario. To match a realistic flight environment of Surveillance UAVs, the UAVs are deployed and flown autonomously across a vast region of $1 \mathrm{Km} \times 1 \mathrm{Km}$. The IEEE 802.11 wireless interface is install on each UAV. The UAV flies 5 minutes above the ground at a speed of 30 meters per second, using a three-dimensional mobility model as illustrated in Figure 7. After establishing a path between the UAV and the base station, the UAV begins transmitting a sensing video with a packet size of 1024 bytes.

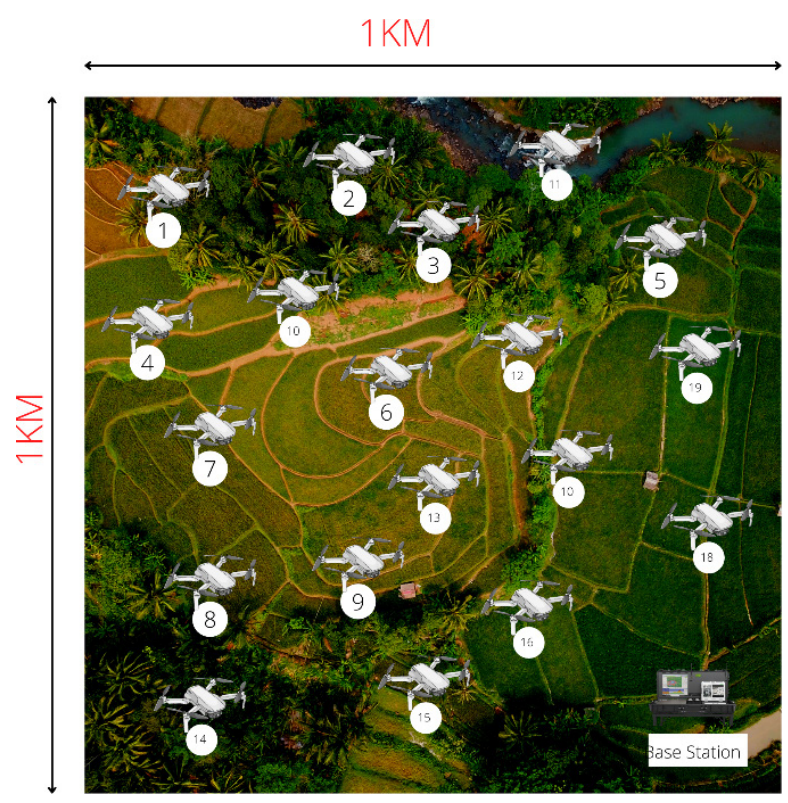

Fig. 7. FANET for surveillance scenario

\subsection{Mobility models 3D}

The Gauss-Markov (GM) mobility model [28] is a three-dimensional time-based model that uses several parameters to respond to varying amounts of randomness and avoid abrupt movement changes. Each movable node is provide a current speed and direction, as illustrated in Figure 8. Based on its previous direction and speed, its upcoming movement is then update and describe. Consequently, GM can eliminate the abrupt pauses and turns seen in Random models. If the right parameters are set, the equations system, which relates prior speed and direction to future ones, enables smooth updating. GM is used to communicate among UAVs in a variety of applications [29]. 


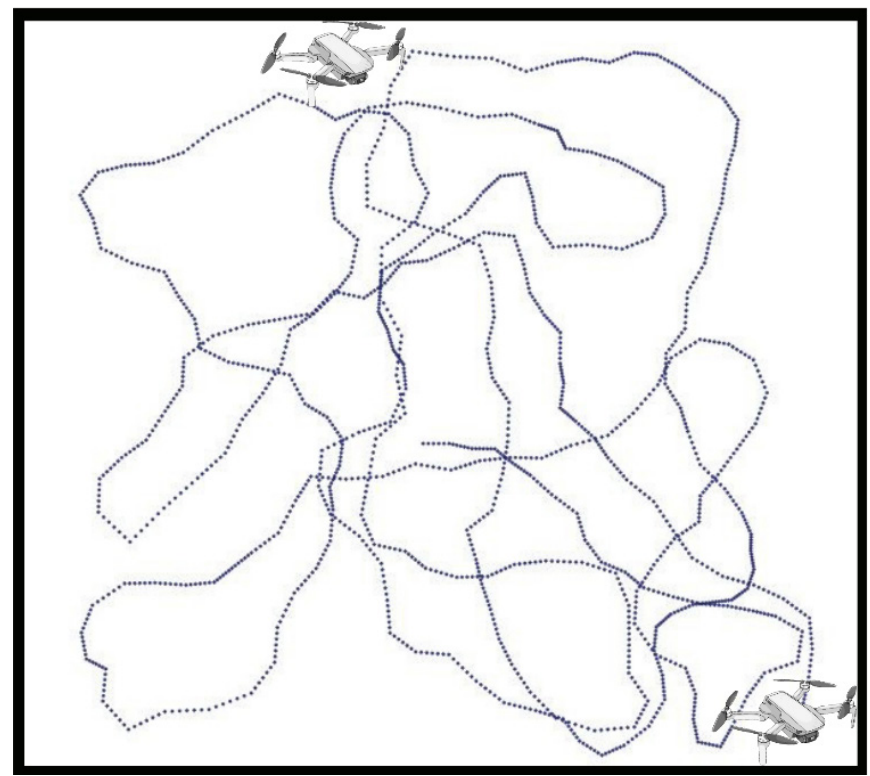

Fig. 8. Trajectory of UAVs using GM models

\subsection{Performance metrics}

The performance of Topology routing protocols analysis and compare based on the following metrics.

- Jitter: The jitter of a packet is measured by the average deviation of the change in packet interval at the receiver compared to the sender, for a pairing of packets, in a flow of packets among a source node and a destination node.

- Average throughput: this metrics represent the total throughput of FANET divide by number of traffic flow for UAVs

- Packet Receive rate: is the rate of the successful received packet by the Base station to the total transmitted packets by the UAV during the mission.

\section{$4 \quad$ Results and discussion}

Figure 9 demonstrate the jitter results of a 20-UAV node for FANET with the a low altitude of $60-150 \mathrm{~m}$ and increments of $10 \mathrm{~m}$. When compared to the other protocols, the OLSR protocol has the lowest jitter. This is owing to its proactive nature and access to the most up-to-date information for all multi-UAV networks. It is also worth noting that when the altitude of UAVs increases, the jitter for all routing protocols goes up as well. Due to its static nature, LCAD, on the other hand, has the greatest Jitter. 


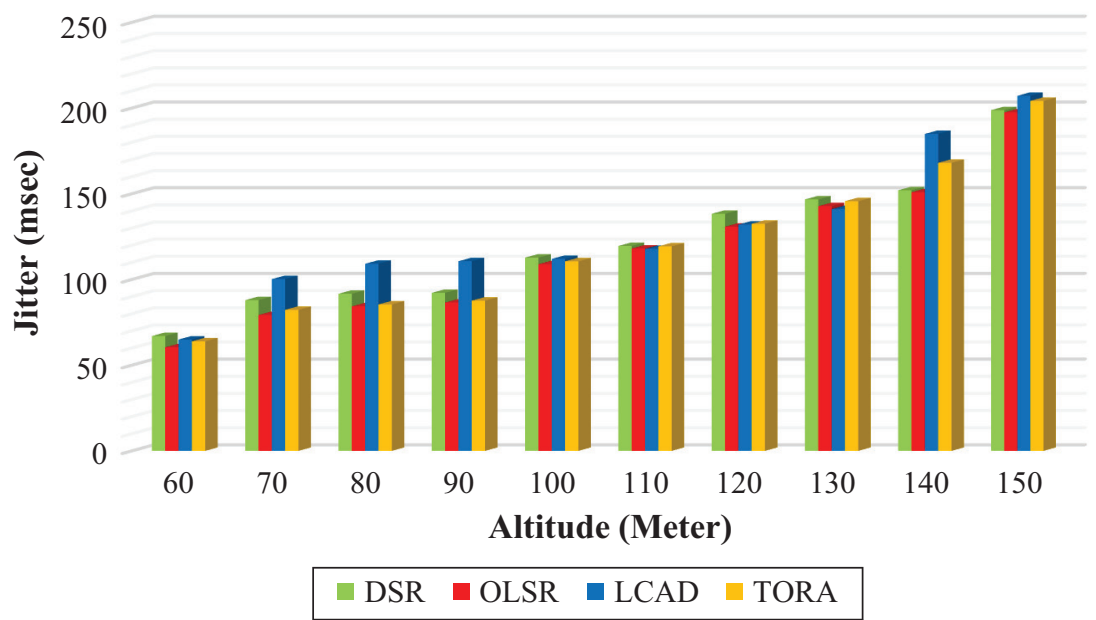

Fig. 9. Jitter comparison

Figure 10 shows a comparison of the average throughput of DSR, OLSR, LCAD, and TORA routing protocols. TORA has the best throughput due to its hybrid nature and effective bandwidth use, whereas LCAD has poor performance in most UAV altitudes. Nevertheless, as the altitude of the UAV increases, the average throughput of all routing protocols drops, particularly beyond 80 meters.

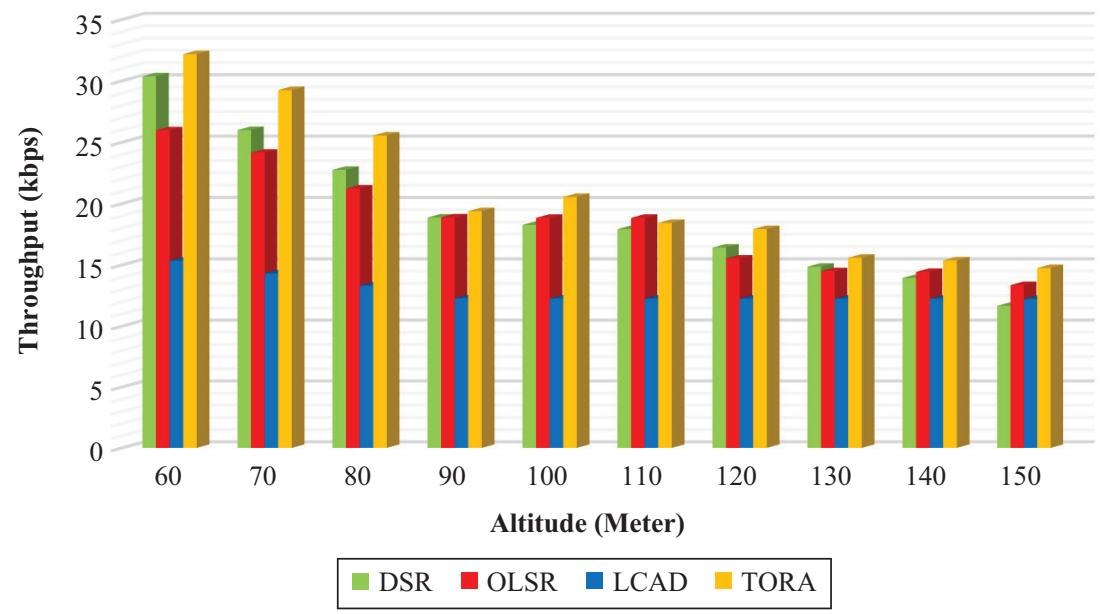

Fig. 10. Average throughput comparison

Figure 11 depicts the packet successful rate of four routing protocols with low altitude for UAVS. It is observed that TORA and DSR protocols provides high reliability for data routing and have highest PSR rate with all UAV Altitudes. Similarly, to other performance metrics LCAD show poor performance and drop many packets. OLSR protocols PSR decrease as the altitude of UAVs increase. 

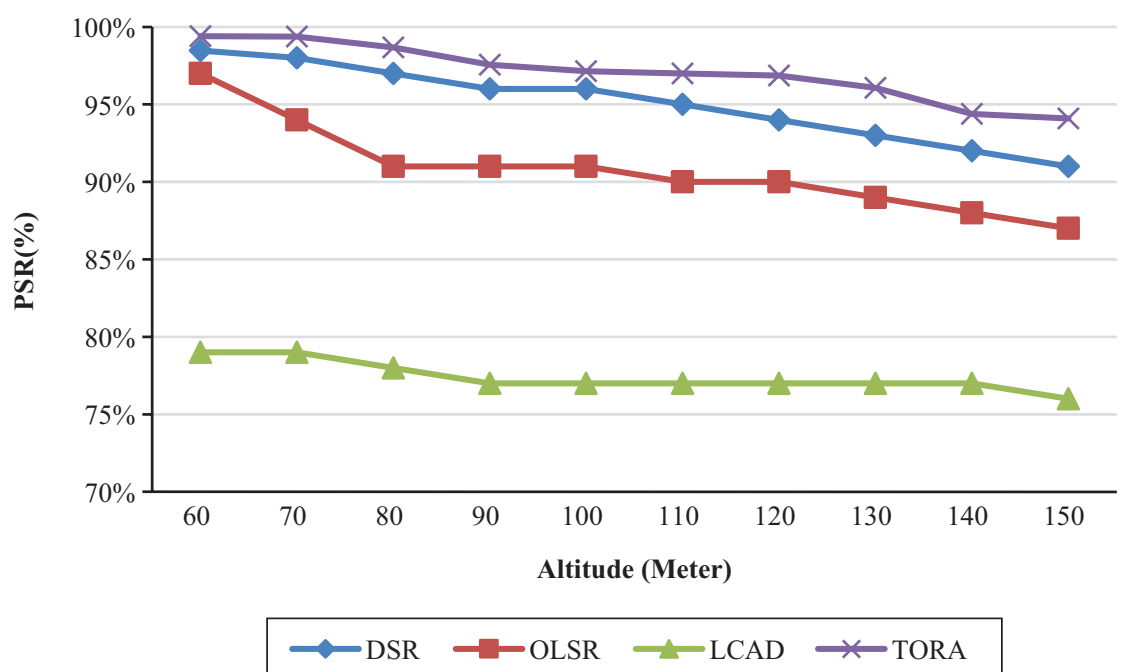

Fig. 11. Packet successful rate comparison

\section{Conclusion}

This research paper investigates the performance of topology-based routing protocols LCAD, DSR, OLSR, and TORA to determine the optimum protocol for surveillance scenarios in Flying Ad hoc Networks. Multiple network metrics, jitter, average throughput, and packet successful rate, were used to analyze and compare the four protocols. In terms of average throughput and DSR, TORA was determined to be the optimal protocol for monitoring scenarios. In terms of overall performance, DSR and OLSR are both ranked second. In simulations, it has been observed that TORA and OLSR performance sometimes goes synonymously and that OLSR jitter delay is sometimes the shortest.

\section{References}

[1] A. Srivastava and J. Prakash, "Future FANET with application and enabling techniques: Anatomization and sustainability issues," Comput. Sci. Rev. Elsevier, vol. 39, p. 100359, 2021. https://doi.org/10.1016/j.cosrev.2020.100359

[2] A. Chriki, H. Touati, H. Snoussi, and F. Kamoun, "FANET: Communication, mobility models and security issues," Comput. Networks, Elsevier, vol. 163, p. 106877, 2019. https:// doi.org/10.1016/j.comnet.2019.106877

[3] M. A. Khan, I. M. Qureshi, and F. Khanzada, "A hybrid communication scheme for efficient and low-cost deployment of future flying ad-hoc network (FANET)," Drones, MDPI, vol. 3 , no. 1, p. 16, 2019. https://doi.org/10.3390/drones3010016

[4] A. R. Ragab, "A new classification for ad-hoc network," Int. J. Interact. Mob. Technol., vol. 14, no. 14, pp. 214-223, 2020. https://doi.org/10.3991/ijim.v14i14.14871 
[5] V. Roberge, M. Tarbouchi, and G. Labonté, "Fast genetic algorithm path planner for fixed-wing military UAV using GPU,” IEEE Trans. Aerosp. Electron. Syst., vol. 54, no. 5, pp. 2105-2117, 2018. https://doi.org/10.1109/TAES.2018.2807558

[6] Z. Kashino, G. Nejat, and B. Benhabib, "Aerial wilderness search and rescue with ground support,” J. Intell. Robot. Syst. Springer, vol. 99, no. 1, pp. 147-163, 2020. https://doi. org/10.1007/s10846-019-01105-y

[7] X. Li and J. Huang, "ABPP: An adaptive beacon scheme for geographic routing in FANET," in 2017 18th International Conference on Parallel and Distributed Computing, Applications and Technologies (PDCAT), IEEE, 2017, pp. 293-299. https://doi.org/10.1109/ PDCAT.2017.00055

[8] A. Bujari, C. E. Palazzi, and D. Ronzani, "FANET application scenarios and mobility models," in Proceedings of the 3rd Workshop on Micro Aerial Vehicle Networks, Systems, and Applications,ACM, 2017, pp. 43-46. https://doi.org/10.1145/3086439.3086440

[9] F. De Rango, G. Potrino, M. Tropea, A. F. Santamaria, and P. Fazio, "Scalable and ligthway bio-inspired coordination protocol for FANET in precision agriculture applications," Comput. Electr. Eng. Elsevier, vol. 74, pp. 305-318, 2019. https://doi.org/10.1016/j. compeleceng.2019.01.018

[10] F. A. Phang et al., "Integrating drone technology in service learning for engineering students," Int. J. Emerg. Technol. Learn., vol. 16, no. 15, pp. 78-90, 2021. https://doi.org/10.3991/ijet. v16i15.23673

[11] M. H. Habaebi, R. K. Omar, and M. R. Islam, "Mobile drone localization in indoor environment based on passive RFID,” Int. J. Interact. Mob. Technol., vol. 14, no. 5, pp. 4-15, 2020. https://doi.org/10.3991/ijim.v14i05.13309

[12] A. Joshi, S. Dhongdi, S. Kumar, and K. R. Anupama, "Simulation of multi-UAV ad-hoc network for disaster monitoring applications," in 2020 International Conference on Information Networking (ICOIN), IEEE, 2020, pp. 690-695. https://doi.org/10.1109/ ICOIN48656.2020.9016543

[13] M. Y.Arafat and S. Moh, "Routing protocols for unmanned aerial vehicle networks: a survey," IEEE Access, vol. 7, pp. 498-516, 2019. https://doi.org/10.1109/ACCESS.2018.2885539

[14] J. Souza, J. Jailton, T. Carvalho, J. Araújo, R. Francês, and Z. Kaleem, "A proposal for routing protocol for FANET: a fuzzy system approach with QoE/QoS guarantee," Wirel. Commun. Mob. Comput., vol. 2019, 2019. https://doi.org/10.1155/2019/8709249

[15] M. F. Khan, K. L. A. Yau, R. M. Noor, and M. A. Imran, "Routing schemes in FANETs: A survey," Sensors ,MDPI., vol. 20, no. 1,pp. 1-33, 2020. https://doi.org/10.3390/s20010038

[16] X. Li, T. Zhang, and J. Li, "A particle swarm mobility model for flying ad hoc networks," in GLOBECOM 2017-2017 IEEE Global Communications Conference, IEEE., 2017, pp. 1-6. https://doi.org/10.1109/GLOCOM.2017.8253966

[17] C. A. Kerrache, E. Barka, N. Lagraa, and A. Lakas, "Reputation-aware energy-efficient solution for FANET monitoring," in 2017 10th IFIP Wireless and Mobile Networking Conference (WMNC),IEEE., 2017, pp. 1-6. https://doi.org/10.1109/WMNC.2017.8248851

[18] J. S. Lee, Y. S. Yoo, H. S. Choi, T. Kim, and J. K. Choi, "Group Connectivity-based UAV positioning and data slot allocation for tactical MANET," IEEE Access, vol. 8, 2020. https:// doi.org/10.1109/ACCESS.2020.3042795

[19] O. S. Oubbati, M. Atiquzzaman, P. Lorenz, M. H. Tareque, and M. S. Hossain, "Routing in flying Ad Hoc networks: Survey, constraints, and future challenge perspectives," IEEE Access, vol. 7, pp. 81057-81105, 2019. https://doi.org/10.1109/ACCESS.2019.2923840

[20] C. M. Cheng, P. H. Hsiao, H. T. Kung, and D. Vlah, "Maximizing throughput of UAV-relaying networks with the load-carry-and-deliver paradigm," IEEE Wirel. Commun. Netw. Conf. WCNC, pp. 4420-4427, 2007. https://doi.org/10.1109/WCNC.2007.805 
[21] A. H. Wheeb and M. T. Naser, "Simulation based comparison of routing protocols in wireless multihop ad hoc networks," Int. J. Electr. Comput. Eng., vol. 11, no. 4, pp. 3186-3192, 2021. https://doi.org/10.11591/ijece.v11i4.pp3186-3192

[22] O. K. Sahingoz, "Networking models in flying Ad-hoc networks (FANETs): Concepts and challenges," J. Intell. Robot. Syst. Theory Appl., vol. 74, no. 1-2, pp. 513-527, 2014. https:// doi.org/10.1007/s10846-013-9959-7

[23] K. Singh and A. K. Verma, "Applying OLSR routing in FANETs," Proc. 2014 IEEE Int. Conf. Adv. Commun. Control Comput. Technol. ICACCCT 2014, IEEE., no. 978, pp. 1212-1215, 2015. https://doi.org/10.1109/ICACCCT.2014.7019290

[24] A. H. Wheeb and N. A. Shiltagh, "Performance analysis of OLSR protocol in mobile ad hoc networks," Int. J. Interact. Mob. Technol., vol. 16, no. 1, 2022. https://doi.org/10.3991/ijim. v16i01.26663

[25] Z. Zheng, A. K. Sangaiah, and T. Wang, "Adaptive communication protocols in flying ad hoc network,” IEEE Commun. Mag., vol. 56, no. 1, pp. 136-142, 2018. https://doi.org/10.1109/ MCOM.2017.1700323

[26] Z. Zhai, J. Du, and Y. Ren, "The application and improvement of temporally ordered routing algorithm in swarm network with unmanned aerial vehicle nodes," Proc. IEEE ICWMC, pp. 7-12, 2013 .

[27] A. H. Wheeb and D. N. Kanellopoulos, "Simulated performance of SCTP and TFRC over MANETs: The impact of traffic load and nodes mobility," Int. J. Bus. Data Commun. Netw., vol. 16, no. 2, pp. 69-83, 2020. https://doi.org/10.4018/IJBDCN.2020070104

[28] D. A. Korneev, A. V Leonov, and G. A. Litvinov, "Estimation of mini-UAVs network parameters for search and rescue operation scenario with Gauss-Markov mobility model," in 2018Systems of Signal Synchronization, Generating and Processing in Telecommunications (SYNCHROINFO), 2018, pp. 1-7. https://doi.org/10.1109/SYNCHROINFO.2018.8457047

[29] N. Lin, F. Gao, L. Zhao, A. Al-Dubai, and Z. Tan, “A 3D smooth random walk mobility model for FANETs," in IEEE 5th International Conference on Data Science and Systems (HPCC/SmartCity/DSS), 2019, pp. 460-467. https://doi.org/10.1109/HPCC/SmartCity/ DSS.2019.00075

\section{$7 \quad$ Author}

Ali H. Wheeb is a Faculty Member - Lecturer at University of Baghdad since 2014. His fields of research interest are flying ad hoc network, mobility models, IoT, wireless ad hoc networking, routing protocols, networking simulation tools Ns-2 \& NS-3, transporting protocols. Further, he publish 11 research papers in high reputation journals. Also. Lecturer Ali serve as reviewer in several journals and conferences and reviewed 100 paper until now. Email: a.wheeb@,coeng.uobaghdad.edu.iq

Article submitted 2021-11-11. Resubmitted 2021-12-17. Final acceptance 2021-12-19. Final version published as submitted by the authors. 